\title{
ANALYTIC EMBEDDING AND MEAN SQUARE APPROXIMATION
}

\author{
SAMUEL MERRILL, III ${ }^{1}$
}

1. Introduction. Our results concern the generalized $H^{p}$ spaces derived from certain function algebras, particularly those spaces associated with analytic discs embedded in the carrier space. Such general $H^{p}$ spaces are obtained by considering a uniform algebra $A=A(X)$, fixing a measure $m$ on $X$ representing a multiplicative linear functional on $A$, and defining $H^{p}(d m)$ as the closure of $A$ in $L^{p}(d m)$ $\left(w^{*}\right.$ closure for $\left.p=\infty\right)$. One further condition (the unique representing measure property) is imposed on $A$ so that a large portion of the classical theory concerning the Hardy classes remains valid for $H^{p}(d m)$.

Our first result provides an explicit determination of the analytic embedding mapping of Wermer [7] (of the unit disc on to the Gleason part associated with $m$ ) in terms of the outer function associated with a certain Radon-Nikodym derivative. We then use this explicit expression to generalize a result of Szegö on mean square approximation of the function 1 by maximal ideals of functions, relating two different homomorphisms in the same Gleason part.

2. Preliminaries. If $X$ is a compact Hausdorff space, a uniformly closed algebra $A=A(X)$ of continuous complex-valued functions is called a uniform algebra if it separates points and contains the constants. A uniform algebra will be called a URM algebra if it also satisfies the following condition isolated by Lumer [5]:

(1) Each multiplicative linear functional $\phi$ on $A$ has a Unique Representing Measure on $X$, i.e., the positive measure $m$ such that $\phi(f)=\int f d m(f \in A)$ is unique.

The most complete exposition for our purposes of the basic results on generalized $H^{p}$ spaces appears in Hoffman [4] where the $H^{p}$ spaces are derived from logmodular algebras. Lumer [5] extended Hoffman's work to URM algebras. For convenience we will refer only to [4] for results of this type.

Presented to the Society, August 31, 1965 under the title $H^{p}$ spaces derived from function algebras; received by the editors September 1, 1966.

1 This research represents one portion of the author's doctoral dissertation at Yale University, where he was a Fellow of the National Science Foundation. The author wishes to express his appreciation to Professor Charles Rickart for his guidance during the preparation of the thesis.

The work was partially supported by NSF grant GP4021 at the University of Rochester. 
Let $A=A(X)$ be a fixed URM algebra. The embedding theorem of Wermer [7] establishes the conjecture of Gleason [2] concerning the analyticity of the Gelfand transform $\hat{f}$ of functions $f$ in $A$ on certain regions of the carrier space (maximal ideal space) $M(A)$, now known as Gleason parts. If we replace the carrier space of homomorphisms by the corresponding space of representing measures on $X$, we may define a Gleason part as an equivalence class of mutually absolutely continuous multiplicative measures. The Radon-Nikodym derivatives relating equivalent measures are bounded. (See Bishop, [1].) To save notation we will use the same symbol to denote a homomorphism of $A$ and its unique representing measure.

For each $f \in H^{p}(d m)$ the Gelfand transform induces a unique extension of $f$ to the part $P(m)$ containing $m$, defined by

$$
\hat{f}(\phi)=\int f d \phi \quad(\phi \in P(m)) .
$$

Suppose that $m$ and $\sigma$ are distinct elements of the same part $P$ of $M(A)$. Let $G_{m}$ be the orthogonal projection of 1 into the subspace $H_{m}^{2}(d \sigma)=\left\{f \in H^{2}(d \sigma): \int f d m=0\right\}$ of $H^{2}(d \sigma)$. Let $Z_{m}$ be the multiple of $G_{m}$ with norm 1 . We now state the theorem of Wermer referred to above.

Theorem (Wermer). For each $f \in H^{2}(d m)$ the equation

$$
\hat{f}(\phi)=F\left(\hat{Z}_{m}(\phi)\right) \quad(\phi \in P)
$$

associates with $f$ a function $F$ analytic on $D=\{|\lambda|<1\}$.

Wermer's proof rests on the observation that since $m$ and $\sigma$ are distinct, it follows from the generalized Beurling Theorem [4, Theorem 5.5, p. 293] applied to the closed invariant subspace $H_{m}^{2}(d \sigma)$ that

$$
H_{m}^{2}(d \sigma)=Z_{m} \cdot H^{2}(d \sigma)
$$

and $\left|Z_{m}\right| \equiv 1$.

REMARK. Relation (3) and the fact that the set $H^{2}(d \sigma)$ does not depend upon the choice of $\sigma$ in $P$ imply that $Z_{m}$ does not depend upon $\sigma$. On the other hand $Z_{m}$ does depend on the choice of $m$, as can be seen from the same relation. For if $Z_{m_{1}}=Z_{m_{2}}$, then $H_{m_{1}}^{2}(d \sigma)=H_{m_{2}}^{2}(d \sigma)$, so that the homomorphisms given by $m_{1}$ and $m_{2}$ have the same kernels in $A$, i.e. $m_{1}=m_{2}$.

Definition. Any function $g \in H^{2}(d m)$ is called outer if it generates $H^{2}(d m)$, i.e., if the set of functions $g h$, where $h$ ranges over $A$, is dense in $H^{2}(d m)$. 
It follows from $\left[4\right.$, Theorem 5.6, p. 294] that $g$ generates $H^{2}(d m)$ if and only if

$$
\int \log |g| d m=\log \left|\int g d m\right|>-\infty .
$$

3. Explicit determination of the embedding function. We now give our explicit expression for the embedding function $Z_{m}$ of Wermer in terms of the Radon-Nikodym derivative relating the measures $m$ and $\sigma$.

Theorem 1. Let $A=A(X)$ be a URM algebra and suppose that $m$ and $\sigma$ are distinct elements of the same part $P$. Write $d \sigma=w d m$ where $w$, $1 / w \in L^{\infty}(d m)$. Then

$$
Z_{m}=(1-g) /(1-k)^{1 / 2}
$$

where $g$ is the unique outer function in $H^{2}(d m)$ for which

(i) $|g|^{2}=k / w\left(k=\exp \int \log w d m\right)$ and

(ii) $\int g d m=1$.

Proof. We apply the generalized Szegö Theorem $[4$, Theorem 4.6, p. 289] to the measure $d \sigma$. It is not difficult to check that $H_{m}^{2}(d \sigma)$ is the closure of the ideal $A_{m}$ of functions in $A$ for which $\int f d m=0$. It follows that $G_{m}$ is the projection of the function 1 in to the closure of $A_{m}$ in $H^{2}(d \sigma)$. Thus by the generalized Szegö Theorem

$$
\begin{aligned}
k & =\inf _{f \in A_{m}} \int|1-f|^{2} w d m=\inf _{f \in H_{m}^{2}(d \sigma)} \int|1-f|^{2} w d m \\
& =\int\left|1-G_{m}\right|^{2} w d m .
\end{aligned}
$$

Writing the second infimum in a different form, we have also

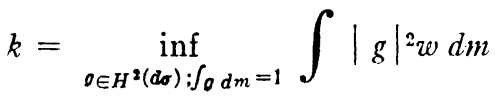

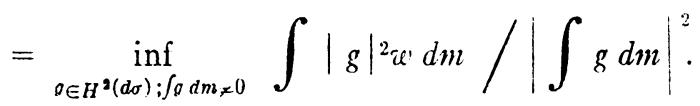

We now show that the function $1-G_{m}$ is outer. To do this let $h$ denote (for the moment) the outer function in $H^{2}(\mathrm{dm})$ for which

$$
|h|=\left|1-G_{m}\right| \text {. }
$$

The existence of such an outer function is insured by [4, Theorem 5.9 , p. 297] since $1-G_{m}$ satisfies the condition 


$$
\int \log \left|1-G_{m}\right| d m \geqq \log \left|\int\left(1-G_{m}\right) d m\right|=0>-\infty .
$$

(The first inequality is the general Jensen Inequality [4, Theorem 5.1, p. 290].) Now using Jensen's Inequality again together with (4)

$$
\begin{aligned}
\left|\int\left(1-G_{m}\right) d m\right|^{2} & \leqq \exp \left(\int \log \left|1-G_{m}\right|^{2} d m\right) \\
& =\exp \left(\int \log |h|^{2} d m\right)=\left|\int h d m\right|^{2} .
\end{aligned}
$$

Thus: $\left|\int\left(1-G_{m}\right) d m\right|^{2} \leqq\left|\int h d m\right|^{2}$. It follows that

(8) $\frac{\int|h|^{2} w d m}{\left|\int h d m\right|^{2}} \leqq \frac{\int\left|1-G_{m}\right|^{2} w d m}{\left|\int\left(1-G_{m}\right) d m\right|^{2}}=\int\left|1-G_{m}\right|^{2} w d m=k$.

In view of (6) we have equality in (8), and in turn equality in (7). That $1-G_{m}$ is outer now follows from (4).

By [4, Theorem 5.8, p. 296],

$$
\left|1-G_{m}\right|^{2} w d m=k d m
$$

so that

$$
\left|1-G_{m}\right|^{2}=k / w .
$$

Since $\int\left(1-G_{m}\right) d m=1, g=1-G_{m}$ is the unique outer function of this modulus which also satisfies condition (ii) of Theorem 1.

To complete the proof we need only show that the constant of normalization is given by $(1-k)^{1 / 2}$. Writing \|\|$_{0}$ for the norm in $H^{2}(d \sigma)$, we have by the general Szegö Theorem

$$
\left|G_{m}\right|^{2}=\left\|G_{m}\right\|_{\sigma}^{2}=1-\left\|1-G_{m}\right\|_{\sigma}^{2}=1-k
$$

Therefore

$$
Z_{m}=(1-g) /(1-k)^{1 / 2} \text {. }
$$

Note that we have in effect proved that the second infimum in (6) need be taken only over the outer functions in $H^{2}(d m)$. This could have been proved directly by applying the argument we used to the general function $g \in H^{2}(d m)$ with mean value nonzero in place of the special function $g=1-G_{m}$. 
4. Extension of Szegö's Theorem. Our second major result, whose proof depends upon the result of Theorem 1, is an extension of the theorem of Szegö on mean square approximation. For the case of the unit disc, Szegö [6] proved in 1920 the prototype for our theorem:

TheOREM (SzEGö). Let $\alpha=r e^{i \theta}$ lie in the interior of the unit disc, and let $A$ be the classical disc algebra. Denote by dm normalized Lebesgue measure and by $P_{r}$ the Poisson kernel. If $\mu$ is a finite positive measure on $|z|=1$ with Lebesgue decomposition $d \mu=h d m+d \mu_{\varepsilon}$, then

$$
\inf _{f \in \boldsymbol{A} ; f(\alpha)=0} \int|1-f|^{2} h d m=\left(1-r^{2}\right) \exp \left(\int \log h(t) P_{r}(\theta-t) d t\right) .
$$

In subsequent generalizations (Helson and Lowdenslager [3] and Hoffman [4]) $A$ is replaced by a URM algebra and $\alpha$ by a point in the carrier space, but the Lebesgue decomposition of $\mu$ is always taken with respect to the measure representing the point $\alpha$. The innovation in our theorem is that the Lebesgue decomposition of $\mu$ is taken with respect to one multiplicative measure $m$ whereas $f$ ranges over an ideal $A_{\sigma}$ corresponding to another multiplicative measure $\sigma$. The context under which our theorem holds occurs precisely when $m$ and $\sigma$ lie in the same analytic disc. We now state and prove our theorem.

Theorem 2. Let $A=A(X)$ be a URM algebra. Suppose $m$ and $\sigma$ lie in the same Gleason part of $M(A)$ and write $d \sigma=w d m$. Let $\mu$ be a positive finite Baire measure on $X$ with $d \mu=h d m+d \mu$. Then

$$
\inf _{f \in A_{\sigma}} \int|1-f|^{2} d \mu=\exp \left(\int \log w d m\right) \cdot \exp \left(\int \log h d \sigma\right) .
$$

Proor. By the Kolmogoroff-Krein addition to the general Szegö Theorem (see Hoffman [4, Theorem 4.3, p. 286]), the measure $\mu$ may be replaced by its absolutely continuous part without altering the infimum. Thus we have, applying the general Szegö Theorem $[4$, Theorem 4.6, p. 289] for the third equality,

$$
\begin{aligned}
& \inf _{\boldsymbol{s} \in \Delta_{\sigma}} \int|1-f|^{2} d \mu=\inf _{f \in A_{\sigma}} \int|1-f|^{2} h d m=\inf _{\boldsymbol{s \in A _ { \sigma }}} \int|1-f|^{2} h / w d \sigma \\
& \quad=\exp \left(\int \log (h / w) d \sigma\right)=\exp \left(\int \log (1 / w)\right) \cdot \exp \left(\int \log h d \sigma\right) .
\end{aligned}
$$

Thus it remains only to show that

$$
\exp \left(\int \log (1 / w) d \sigma\right) \cdot \exp \left(\int \log (1 / w) d m\right)=1
$$


By Theorem 1

$$
w=k /|g|^{2}
$$

where $g$ is an outer function in $H^{2}(d m)$ and $k=\exp \left(\int \log w d m\right)$ as before. Thus (11) becomes

$$
\exp \left(\int \log |g|^{2} d \sigma\right) \cdot \exp \left(\int \log |g|^{2} d m\right)=k^{2} .
$$

Now observe that the $L^{2}(d \phi)$ closure of the functions $\{g h: h \in A\}$ is the same for all $\phi$ lying in the Gleason part containing $m$ and $\sigma$. Therefore by the definition of outer function, $g$ is outer with respect to every $\phi$ in this part. Thus $g$ satisfies (4) for both $m$ and $\sigma$, so that (12) is replaced in turn by

$$
\left|\int g d \sigma\right| \cdot\left|\int g d m\right|=k .
$$

By Theorem 1, $\int g d m=\int\left(1-G_{m}\right)=1$. To complete the proof, it suffices to show that $\int g d \sigma=k$. But by the definition of $G_{m}$ and (9)

$$
0=\int G_{m}\left(1-\bar{G}_{m}\right) d \sigma=\int G_{m} d \sigma-\int\left|G_{m}\right|^{2} d \sigma=\int G_{m} d \sigma-(1-k) .
$$

Therefore $\int g d \sigma=\int\left(1-G_{m}\right) d \sigma=1-(1-k)=k$.

CoRollary 1. Property (i) which appears in the statement of Theorem 1 may be replaced by

$$
|g|^{2}=k^{\prime} v
$$

where $v$ is defined by $d m=v d \sigma($ i.e. $v=1 / w)$ and $k^{\prime}=\exp \left(\int \log v d \sigma\right)$.

Proof. That $k^{\prime}=k$ follows directly from item (11) of the proof of Theorem 2. Note that (11) states that the geometric mean relating two measures of the same Gleason part is the same in either direction.

COROLlary 2. Under the hypothesis of Theorem 2

$$
\inf _{f \in A_{\sigma}} \int|1-f|^{2} d m=\inf _{f \in A_{m}} \int|1-f|^{2} d \sigma .
$$

Proof. The left hand-side is seen to equal $\exp \left(\int \log w d m\right)$ by setting $d \mu=d m$ in Theorem 2. This geometric mean also equals the right-hand side by the general Szegö Theorem.

\section{REFERENCES}

1. E. Bishop, Representing measures for points in a uniform algebra, Bull. Amer. Math. Soc. 70 (1964), 121-122. 
2. A. Gleason, "Function algebras," in Seminars on analytic functions, Vol. II, Inst. for Advanced Study, Princeton, N. J., 1957.

3. H. Helson and D. Lowdenslager, Prediction theory and Fourier series in several variables, Acta Math. 99 (1958), 165-202.

4. K. Hoffman, Analytic functions and logmodular Banach algebras, Acta Math. 108 (1962), 271-317.

5. G. Lumer, Analytic functions and Dirichlet problem, Bull. Amer. Math. Soc. 70 (1964), 98-104.

6. G. Szegö, Beiträge zur Theorie der toeplitzen Formen (Ersten Mitteilung), Math. Z. 6 (1920), 167-202.

7. J. Wermer, Dirichlet algebras, Duke Math. J. 27 (1960), 373-382.

YALE UNIVERSITY AND

THE UNIVERSITY OF ROCHESTER 